\title{
Percepção de estagiários da área da saúde e trabalhadores de Instituições de Longa Permanência de Idosos sobre a institucionalização
}

\author{
Perception of health trainees and workers from Long-Term \\ Care Institutions for the Elderly on institutionalization
}

Fernanda dos Santos Pascotini1 ${ }^{1}$, Elenir Fedosse $e^{1,2,3}$

'Programa de Pós-Graduação em Distúrbios da Comunicação Humana, Universidade Federal de Santa Maria (UFSM) - Santa Maria (RS), Brasil. 2Programa de Residência Multiprofissional Integrada em Sistema Público de Saúde, (UFSM) - Santa Maria (RS), Brasil.

${ }^{3}$ Programa de Pós-Graduação em Gerontologia (UFSM) - Santa Maria (RS), Brasil.

DOI: http://dx.doi.org/10.7322/abcshs.v43i2.1026

\begin{abstract}
RESUMO
Introdução: Com o progressivo aumento do envelhecimento da população, a institucionalização cresce, especialmente por, na maioria das vezes, ser a única opção de moradia e cuidado para idosos e/ ou seus familiares. As concepções dos profissionais que atuam nas instituições repercutem em seus comportamentos e formas de atender/ cuidar em saúde. Objetivo: Identificar a percepção de estagiários da área da saúde e trabalhadores de Instituição de Longa Permanência para Idosos (ILPI) sobre a institucionalização. Métodos: Estudo qualiquantitativo através de questionário preenchido pelos estagiários e trabalhadores da ILPI. Dados interpretados a partir da análise descritiva dos dados e análise de conteúdo. Resultados: 40 indivíduos participaram da pesquisa, sendo $65 \%$ estagiários da saúde e $35 \%$ trabalhadores. Quanto aos aspectos positivos da institucionalização, surgiram duas categorias: assistência emocional (apoio e segurança, carinho e socialização entre os idosos) e assistência física (cuidados especializados e acessibilidade). Quanto aos aspectos negativos, surgiram três categorias: distanciamento do mundo exterior (afastamento da família, de bens materiais e isolamento social), regras impostas: rotina da instituição, falta de privacidade/liberdade e ausência de lazer; cuidado profissional: falta de especialidades e despreparo de profissionais. Quando questionados se morariam em uma ILPI na velhice, 22 afirmaram que sim. Conclusão: Surge a necessidade do seguimento do estudo, sugerindo-se estratégias capazes de minimizar os aspectos negativos da institucionalização nesta ILPI, detectados pela amostra, e maximizar/melhorar os aspectos positivos. É papel dos trabalhadores aprimorarem-se continuamente para melhorar as condições de funcionamento das instituições em que trabalham.
\end{abstract}

Palavras-chave: idoso; institucionalização; envelhecimento; padrão de cuidado.

\begin{abstract}
Introduction: With the increasing aging of the population, institutionalization expands, especially since, for the most part, it is the only option of housing and care for the elderly and/or their families. The conceptions of the professionals that work in institutions have repercussions on their behaviors and ways of attending/caring for health. Objective: To identify the perception of health trainees and workers from geriatric long-term care facilities (ILPI) about institutionalization. Methods: Qualitative and quantitative study through a questionnaire filled out by ILPI trainees and workers. Data interpreted from descriptive data analysis and content analysis. Results: 40 individuals participated in the research, $65 \%$ of which were health trainees and $35 \%$ were workers. As for the positive aspects of institutionalization, two categories emerged: emotional assistance (support and security, caring and socialization among the elderly) and physical assistance (specialized care and accessibility). As for the negative aspects, three categories emerged: estrangement from the outside world (detachment from the family, material goods and social isolation), imposed rules (routine of the institution, lack of privacy/freedom and absence of leisure) and professional care (lack of specialties and unprepared professionals). When asked whether they would live in a ILPI in old age, 22 answered yes. Conclusion: There is a need to follow up the study, suggesting strategies capable of minimizing the negative aspects of institutionalization in this ILPI, detected by the sample, and maximizing/improving the positive aspects. It is the role of workers to continually improve themselves to improve the working conditions of the institutions in which they work.
\end{abstract}

Keywords: aged; institutionalization; aging; standard of care.

Autor para correspondência: Fernanda dos Santos Pascotini - Centro de Ciências da Saúde - Avenida Roraima, 1000 - Cidade Universitária - CEP: 97105 -900 Santa Maria (RS), Brasil - E-mail: fepascotini@hotmail.com

Conflito de interesses: nada a declarar 


\section{INTRODUÇÃO}

O aumento do número de idosos é uma situação contemporânea em países desenvolvidos ou em processo de desenvolvimento. No Brasil, este fato acontece aceleradamente, principalmente pela redução dos números de fecundidade e pelo acréscimo significante da expectativa de vida da população ${ }^{1}$, que aumentou para 73,1 anos, com projeção de alcançar a média de 81,2 anos em $2050^{2}$. Nas próximas duas décadas, a população idosa brasileira representará $14 \%$ da população total e até 2050 esse valor irá triplicar, chegando aos 65 milhões ${ }^{3}$.

Com o envelhecimento da população, a institucionalização de idosos tem aumentado, por motivos diversos, como: 1) mudança no número de pessoas e na constituição das famílias, aliada à inserção da mulher ao mercado de trabalho, já que no passado, era a responsável pelos cuidados dos mais velhos ; 2) dificuldades das famílias em acolhê-los (por falta de espaço, recursos, estrutura familiar ou por incapacidade de cuidar, de localizar um cuidador/ acompanhante, baixa renda, viúvo) e 3) opção do idoso por pensar ser um peso para sua família. Muitas vezes, residir em uma instituição de longa permanência para idosos (ILPI) é a única opção para o sujeito e seus familiares, fato que provoca grandes mudanças do estilo de vida e do projeto existencial do idoso 5 .

Reconhecidamente, o envelhecimento representa um processo biopsicosociocultural que gera demandas complexas, exigindo, pois, um cuidado diferenciado dos profissionais da saúde $e^{6,7}$. Sabese que as concepções/crenças dos profissionais repercutem seus comportamentos e, consequentemente, suas formas de atender/ cuidar em saúde ${ }^{7}$, por isso, convém investigar a compreensão que os profissionais de saúde têm acerca da institucionalização. A partir dos resultados, pode-se detectar as qualidades e as deficiências de uma instituição, observados pelo ângulo de quem não reside, apenas trabalha, podendo servir para estabelecimento de ações, com o objetivo de aprimorar seu funcionamento e favorecer a qualidade de vida dos idosos.

Sendo assim, o objetivo desta pesquisa foi identificar a percepção dos estagiários graduandos na área da saúde e também dos trabalhadores de uma ILPI filantrópica de um município gaúcho de médio porte sobre a institucionalização.

\section{MÉTODOS}

Estudo de campo/exploratório, de abordagem quali-quantitativa, aprovado pelo Comitê de Ética em Pesquisa com Seres Humanos da Universidade Federal de Santa Maria- RS (0324.0.243.000-11), realizado com estagiários graduandos em Enfermagem, Fisioterapia, Fonoaudiologia e trabalhadores (enfermeira, psicóloga, fisioterapeuta, assistente social, administradora, técnicas em enfermagem, cuidadora e serviços gerais) que atuam nesta ILPI.

O campo de pesquisa foi uma ILPI de natureza filantrópica, localizada em um município do Rio Grande do Sul. Trata-se de uma Instituição fundada em 1949, mantida por doações da sociedade em geral e comunidade religiosa a que pertence percentual legal da aposentadoria dos residentes e projetos ou campanhas governamentais. Reside nessa ILPI, cerca de trinta e um idosos, a maioria do sexo feminino, com idades que variam entre 60 e 90 anos. Conta com dezenove profissionais, quatorze deles participantes desta pesquisa. A assistência à saúde dos idosos é ampliada por meio de convênios com instituições de ensino médio profissionalizante e superior, contando com aproximadamente 40 estagiários, sendo 26 participantes desta pesquisa.

Foram incluídos os estagiários graduandos da saúde e trabalhadores da ILPI (acima identificados), atuantes há mais de um mês e que estavam presentes no período da coleta (01 a 25 de outubro de 2016).

Os estagiários e trabalhadores foram convidados a participarem da pesquisa, firmaram o Termo de Consentimento Livre e Esclarecido e responderam um questionário com dados pessoais (sexo, idade e função na ILPI) e duas questões abertas: uma sobre os aspectos positivos e negativos da institucionalização (na sua opinião, quais os aspectos positivos e quais os aspectos negativos da institucionalização?) e a outra de cunho pessoal/projeção da velhice (você moraria em uma ILPI? Justifique).

Para atingir os objetivos deste estudo, foi utilizado o método "Análise de Conteúdo", que indica encontrar respostas para as perguntas estabelecidas e, além do mais, tem relação com o descobrimento dos elementos próprios ao conteúdo aparente. A partir da análise de conteúdo, o tratamento dos dados deu-se em três fases: a primeira de pré-análise (seleção e organização do conteúdo) a ser considerado, composto pelas tarefas: leitura flutuante, constituição do Corpus e formulação de Hipóteses e objetivos; a segunda fase é da exploração do material que se destina a realizar a codificação, período onde se deve dedicar-se ao que ficou definido na fase anterior, podendo ser necessárias muitas leituras de um mesmo material. Em uma terceira fase, realizamos o tratamento do que foi obtido e sua interpretação, com vista em expor o conteúdo que está subentendido no manifestado, sem esquecer-se das informações estatísticas, devendo-se voltar para ideologias e intenções que propõem os fenômenos ponderados.

Foram criadas cinco categorias para a primeira questão (aspectos positivos: assistência física e emocional; aspectos negativos: distanciamento do mundo exterior, imposição de regras e cuidado profissional insuficiente) e duas para a segunda (sim e não com respectivas justificativas).

\section{RESULTADOS E DISCUSSÃO}

Participaram da pesquisa, 40 pessoas, sendo: 65\% (26) estagiários graduandos na área da saúde e 35\% (14) trabalhadores da ILPI. Nota-se que em relação ao gênero e a idade, 92,5\% (37) eram mulheres com idade média de 30,7 anos; 7,5\% (3) de homens com 38,7 anos em média de idade. A média geral de idade dos participantes, independentemente do gênero, foi de 31,3 anos, sendo o mais novo com 20 e o mais velho, 62 anos. 
Constatou-se, neste estudo, tal como em outros ${ }^{9,10}$, o predomínio do gênero feminino no ato de cuidar. Segundo os autores referenciados, tal fato explica-se pela relação da mulher com seus filhos, facilitando sua adaptação ao cuidado de idosos. Tal processo é denominado de feminização do cuidado e pode contribuir para maior humanização das ILPI.

Quando questionados sobre os aspectos positivos da institucionalização, pode-se elaborar, conforme indicado, duas categorias: assistência emocional e assistência física.

A categoria assistência emocional englobou os seguintes aspectos: 1) possibilidade de socialização entre os idosos (sendo referida por 18 dos participantes, 77,8\% dos estagiários e 22,2\% dos trabalhadores); 2) apoio e segurança do idoso (relatada por 5 participantes, $80 \%$ dos estagiários e $20 \%$ dos trabalhadores) e oportunidade de receber carinho (referida por dois estagiários participantes da pesquisa). A propósito, uma pesquisa realizada com 10 idosos de uma ILPI, do noroeste do RS, revelou que, na percepção deles, o apoio e a segurança oferecida no ambiente institucional, são os principais fatores de bem estar e felicidade ${ }^{11}$. Neste estudo, ficou evidente que os estagiários e trabalhadores da instituição têm forte relação com os idosos. Assim, os pontos positivos apontados têm a ver com os sentimentos da equipe da ILPI em relação aos idosos, gerando prestação de cuidado comprometido com a segurança e bem-estar dos idosos.

Estudos revelam ${ }^{11,12}$ que o idoso institucionalizado sente-se excluído, à medida que perde contato com parentes/ambiente familiar. Com isso, vivencia novas formas de adaptação no ambiente institucional, sendo o afastamento familiar suprido pelo contato afetuoso com outros idosos e com a equipe de profissionais. Estudo ${ }^{13}$ sugere que uma boa estratégia para melhorar o bem-estar dos idosos nas ILPI é observar o vínculo entre eles e incentivar o agrupamento considerando as afinidades pessoais. Outro estudo ${ }^{14}$ revela que o desenvolvimento de vínculos de afeto entre os trabalhadores da ILPI e seus residentes proporciona prazer e, conforme essas junções vão aumentando, ocorre uma troca de relação profissional por um relacionamento familiar.

A categoria - assistência física - incluiu cuidados especializados em saúde e acessibilidade. A oferta de cuidados especializados foi apontada como aspecto positivo por 21 participantes da pesquisa, sendo $57,1 \%$ dos estagiários e $42,9 \%$ dos trabalhadores da ILPI. Sabe-se que as ILPI assumem a responsabilidade do cuidado em saúde quando o idoso perde vínculo com sua vida social, assistindo e superando suas precisões, a fim de aumentar sua qualidade de vida ${ }^{15}$. O cuidado ofertado aos idosos de acordo com as necessidades nos diversos níveis de dependência que precisam e, ainda, conseguem realizarem programas voltados à redução e prevenção de morbidades ${ }^{16}$.

Estudo com o objetivo de identificar o nível de satisfação de 23 idosos residentes e de oito profissionais de enfermagem de uma ILPI, no Paraná, com relação ao cuidado prestado, identificou que a assistência realizada pelo grupo de enfermagem deve envolver conhecimentos antecipados de todos os moradores e de suas particularidades para que se possa oferecer um cuidado harmônico com as condições individuais, a fim de que não sobrevenha nenhuma interferência na relação profissional/idoso. $\mathrm{Na}$ avaliação do grupo etário tratado, os profissionais possuem criatividade e capacidades suficientes para o estabelecimento de vínculos, o que favorece o cuidado humanizado, comunicação efetiva e obtenção de reações positivas dos mesmos. Para os profissionais, a criação de vínculo é valiosa, visto que facilita a contribuição da assistência e, assim, pode acarretar nível maior de contentamento dos profissionais com o serviço que prestam e dos idosos que ganham a assistência ${ }^{17}$.

A acessibilidade foi citada por três trabalhadores da ILPI como positiva na institucionalização. A Resolução $n^{\circ} .283$ da Agência Nacional de Vigilância Sanitária (Anvisa) ${ }^{18}$ aprovou o Regulamento Técnico que define normas de funcionamento para as ILPI e determina que as secretarias de saúde estaduais, municipais e do Distrito Federal precisam implementar métodos para sua adoção. Pode-se dizer que a baixa indicação da acessibilidade está relacionada ao fato de a ILPI, deste estudo, estar devidamente acessível há vários anos, condição então já naturalizada para os trabalhadores.

Com relação aos aspectos negativos da institucionalização, surgiram três categorias: distanciamento do mundo exterior imposição de regras e cuidados profissionais insuficientes. $\mathrm{Na}$ categoria distanciamento do mundo exterior, destacaram-se os seguintes itens: afastamento da família, afastamento de bens materiais e isolamento social.

O afastamento da família foi indicado por 23 dos pesquisados (sendo 65,2\% estagiário e 34,8\% trabalhadores). Portanto, a percepção dos participantes desta pesquisa coincide com a literatura produzida referente à relação idoso institucionalizado e relação familiar, conforme argumentação que se segue.

A convivência em família é uma experiência abastada em oportunidades para a construção de diferentes relacionamentos pessoais; na família ocorre a troca de necessidades, dificuldades, capacidades e incapacidades relacionais de todos e de cada um ${ }^{19,20}$. E, como nem sempre a família pode tomar para si a responsabilidade no cuidado do idoso em sua casa, tanto por problemas socioeconômicos como por falta de estrutura, recorre à institucionalização, considerando-a como local que pode proporcionar os cuidados adequados às necessidades do idoso ${ }^{21}$. A situação de fragilidade do idoso, sua perda de autonomia e independência e/ ou o afastamento dos vínculos afetivos familiares também podem ser causas da institucionalização ${ }^{22,23}$.

Estudos ${ }^{23,24}$ afirmam que o papel da família é de suma importância para o idoso e não está relacionado somente ao ato de cuidar, mas especialmente ao afeto que pode ofertar. Portanto, o vínculo com a família é algo que deve ser preservado após a institucionalização. Outros estudo ${ }^{23,25}$ relataram que idosos com depressão atribuíram-na pela ausência dos familiares.

O distanciamento da família pode agravar a trajetória de vida do idoso, então acredita-se que saber sobre o vínculo familiar e a 
relação dele, quando na admissão do idoso na instituição, pode colaborar em intervenções que tendem a reduzir situações de conflito entre o idoso e familiares, assim como abreviar sua fragilidade ao desenvolvimento de patologias como a depressão ${ }^{13}$.

Pesquisa realizada na Espanha revelou que a sustentação da independência do idoso em ILPI durante um ano teve muita relação com o suporte familiar recebido, visto que idosos com este apoio apresentavam maior autonomia do que aqueles que não contavam com este tipo de ajuda ${ }^{26}$. Na Colômbia, pesquisadores observaram que as visitas dos filhos ou outros membros da família eram importantes para a melhora do contentamento dos idosos residentes em ILPI $^{27}$.

Nessa pesquisa, 3 dos participantes ( 1 estagiário e 2 trabalhadores) relataram como aspecto negativo da institucionalização, o afastamento de bens materiais. Vale lembrar que quando o idoso vai para uma ILPI, ele deixa seus pertences e familiares e necessita de uma adaptação à nova realidade, com pessoas distintas e à rotina de um ambiente diferente, que muitas vezes não oferece condições favoráveis para uma vida digna ${ }^{23}$. Quando o idoso deixa a sua própria casa, deixa de lado seus bens pessoais, mas também significados de uma vida inteira ${ }^{28}$. Os estudos de Rissardo et al. ${ }^{13}$ e Rocha et al. ${ }^{29}$ sugere que o procedimento de (re)organização da vida do idoso pode ser auxiliado pela ILPI, quando lhe é autorizado manter ligações com sua história de vida. Portanto, sugere-se que o idoso possa manter seus objetos pessoais, para que o ambiente tenha um toque de maior identidade, mais familiar e aconchegante. Atos neste âmbito podem favorecer a melhora da qualidade de vida dos idosos institucionalizados, a reorganização do ambiente é critério fundamental para a satisfação do idoso.

Outro aspecto apontado como negativo por nove dos participantes desta pesquisa (66,7\% estagiários e $33,3 \%$ trabalhadores) foi o isolamento social. $\mathrm{O}$ processo do envelhecimento, a rede social do idoso diminui, principalmente por causa do declínio funcional característico deste estágio da vida, como a limitação física para se deslocar, passando a maior parte de seu tempo só ${ }^{30}$. O comportamento de isolamento social acarreta uma série de repercussões na saúde do idoso e cabe aos profissionais das ILPI, incentivar relações de amizade, promovendo sua saúde e fazendo-o experimentar o prazer da criação de novos vínculos em circunstâncias diferentes das então vivenciadas ${ }^{13}$.

A rotina estabelecida pela ILPI, a falta de privacidade/liberdade e a ausência de lazer foram aspectos referidos pelos participantes deste estudo - incluídos na categoria intitulada "regras impostas".

A rotina determinada pela ILPI foi considerada como aspecto negativo para 7 profissionais $(85,7 \%$ estagiários e $14,3 \%$ trabalhadores). Sabe-se que cada ILPI possui suas normas, procedimentos e rotinas, estabelecidas pelo seu responsável e, impostas para todos seus moradores, desconsiderando-se as diferenças individuais e a história de vida de cada um. Argumenta-se que as regras estabelecidas pelas ILPI, apesar de não agradar a maioria dos idosos, são indispensáveis para manter a organização em um local de habitação coletiva ${ }^{17}$. Existem, atualmente, instituições que seguem os padrões estabelecidos pelo Ministério da Saúde que recomenda uma assistência individualizada aos idosos, mas ainda são minoria ${ }^{23,31}$.

A falta de privacidade/liberdade foi indicada por 6 participantes $(66,6 \%$ estagiários e $33,4 \%$ trabalhadores) e corrobora com estudo $^{13}$ que revelou a falta de liberdade como o fator de maior aborrecimento dos idosos institucionalizados, que se sentem incomodados por terem que se alimentar nos horários determinados, manterem contatos não apetecidos, participarem de atividades que não lhes proporciona contentamento e saírem da instituição somente com a liberação de um responsável.

O idoso residente em ILPI tem que compartilhar sua vida com pessoas desconhecidas sendo obrigado a adaptar seus hábitos, horários, dietas e atividades impostas pela instituição ${ }^{26}$. É frequentemente vigiado para evitar que desobedeçam as regras, costumes e limites estabelecidos pela instituição ${ }^{16}$. Assim, fica muito difícil para o idoso ter seu espaço particular, o que pode proporcionar a solidão, tristeza profunda e afastamento pela falta de individualidade pessoal, vida social, afetiva e sexual, além de falta de esperança no futuro ${ }^{13}$.

Ao lado dos aspectos acima discutidos, foi apontada por 2 indivíduos, a ausência de lazer (1 estagiário e 1 trabalhador). Esta consideração pode estar assentada na perspectiva de que a velhice se distingue de outras etapas da vida em função do tempo disponível que dispõe. Estudo ${ }^{13}$ afirma que se o tempo livre não for aproveitado de forma adequada, pode contribuir com o afastamento, à tristeza, à redução de identidade e à baixa autoestima. Por isso, sugere que as ILPI tenham um planejamento de atividades que visem à ocupação dos idosos (oficinas artesanais, contato com o mundo externo, como viagens, passeios, entre outros). Outro $^{16}$ recomenda que as ILPI ofereçam oportunidades para que a sociedade possa visitar suas dependências, organizando atividades que propiciem o contato tanto de pessoas mais jovens como de outros idosos com os residentes.

A falta de algumas especialidades profissionais da área da saúde foi apontada por três dos participantes desta pesquisa (dois estagiários e um trabalhador), bem como o despreparo de alguns profissionais (citado por três profissionais, dois estagiários e um trabalhador).

Possivelmente, os participantes que indicaram a escassez de profissionais assumem a concepção de que o cuidado ao idoso institucionalizado necessita de abordagem multiprofissional, fazendo-se preciso o envolvimento de um conjunto de trabalhadores, possibilitando troca de saberes, para oferecer um atendimento integral e adequado às necessidades dessa população. Estudo ${ }^{16}$ revelou que, ainda existem ILPI com trabalhadores com pouca ou nenhuma capacitação na área de gerontologia, em número pequeno, com baixo salário e com acúmulo de funções. A propósito, o despreparo de alguns profissionais também foi destacado como ponto negativo da institucionalização, o que corrobora com a interpretação de que há necessidade premente de capacitar o profissional de saúde que trabalha com idosos no Brasil ${ }^{32}$. 
A maioria das ILPI brasileiras é filantrópica e conta com o apoio de congregações religiosas da comunidade. Desta forma, as ILPI encontram poucos recursos, principalmente com relação a pessoal qualificado/capacitado para o cuidado de idosos ${ }^{33}$. Assim, é mais frequente que as atividades laborais sejam realizadas por auxiliares/técnicos em enfermagem e/ou cuidadoras que estiveram ou ainda são os responsáveis pelos serviços gerais ${ }^{34}$. Estudo recente investigou a formação profissional de 63 cuidadores atuantes em nove ILPI do Rio Grande do Norte, concluindo, no geral, uma inadequada capacitação formal para o exercício do cuidado, mostrando deficiências na formação, relacionadas à inexistência de um currículo básico e a baixa escolaridade requisitada ${ }^{35}$.

As situações acima destacadas contrariam a Política Nacional de Saúde da Pessoa Idosa, instituída pela Portaria nº 2.528 , de outubro de 2006, que determina que as atividades para o cuidado aos idosos determinam um tratamento integral, interdisciplinar e multidimensional, sempre considerando a interação dentre os fatores físicos, psicológicos e sociais que afetam sua saúde, bem como a importância do meio em que estão imersos ${ }^{36,37}$.

Quanto à segunda questão: você moraria em uma ILPI na sua velhice - 22 dos participantes afirmaram que sim (45,4\% estagiários e $54,6 \%$ trabalhadores); três (13,7\%) relataram que morariam em função da assistência recebida e por satisfação e autonomia, ou seja, para não atrapalhar a família em seu cotidiano, que pode ser diferente de suas necessidades de idoso. Os outros 19 (86,3\%) participantes que morariam em ILPI, afirmaram que somente iriam por necessidade financeira, de saúde ou impossibilidade de a família mantê-los em seu meio.
Já os 18 que relataram não desejar residir em ILPI na velhice $88,9 \%$ estagiários e $11,1 \%$ trabalhadores, destacaram que a família tem obrigação de cuidar de seus idosos, especialmente, como forma de gratidão pelo esforço que fizeram durante a vida para mantê-la.

Conclui-se que, quanto aos aspectos positivos da institucionalização, surgiram duas categorias: assistência emocional (apoio e segurança, carinho e socialização entre os idosos) e assistência física (cuidados especializados e acessibilidade). Quanto aos aspectos negativos da institucionalização, surgiram três categorias: distanciamento do mundo exterior (afastamento da família, afastamento de bens materiais e isolamento social), regras impostas pela instituição (rotina, falta de privacidade/liberdade e ausência de lazer) e cuidado profissional insuficiente (falta de algumas especialidades profissionais da área da saúde e despreparo de alguns profissionais).

Quanto à motivação dos participantes em morarem ou não em uma ILPI, obteve-se maior percentual relativo à institucionalização, no entanto, os motivos que a sustentam foram impedimentos por necessidade financeira, de saúde ou impossibilidade de a família mantê-los em seu meio e não opção voluntária.

Sendo assim, surge aos autores a necessidade da continuação deste estudo, sugerindo-se estratégias que possam minimizar os aspectos negativos da institucionalização nesta ILPI, detectados pela amostra e maximizar/melhorar os aspectos positivos. É papel dos profissionais da saúde aprimorarem-se continuadamente para melhorar as condições de funcionamento das instituições em que trabalham.

\section{REFERÊNCIAS}

1. Soares LDA, Campos FACS, Araújo MGR, Falcão APST, Lima BFDA, Siqueira DF, et al. Análise do desempenho motor associado ao estado nutricional de idosos cadastrados no Programa Saúde da Família, no município de Vitória de Santo Antão-PE. Ciênc Saúde Coletiva. 2012;17(5):1297-1304. http://dx.doi.org/10.1590/S1413-81232012000500023

2. Instituto Brasileiro de Geografia e Estatística (IBGE). Notícias Censo 2010. IBGE; 2010

3. Andrade LM, Sena ELS, Pinheiro GML, Meira EC, Lira LSSP. Políticas públicas para pessoas idosas no Brasil: uma revisão integrativa. Ciênc Saúde Coletiva. 2013; 18(12):3543-52. http://dx.doi.org/10.1590/S1413-81232013001200011

4. Watanabe HAW, VM. Instituições de Longa Permanência para Idosos (ILPI). Bol Inst Saúde. 2009;(47):69-71.

5. Borges MGS, Rocha LR, Couto EAB, Mancini PC. Comparação do equilíbrio, depressão e cognição entre idosas institucionalizadas e não-institucionalizadas. Rev CEFAC. 2013;15(5):1073-9. http://dx.doi.org/10.1590/S1516-18462013000500003

6. Perez EA. Enfermeria gerontologica: conceptos para la práctica. Washington: OPAS; 1993
7. Schimidt TCG, Silva MJP. Percepção e compreensão de profissionais e graduandos de saúde sobre o idoso e o envelhecimento humano. Rev Esc Enferm USP. 2012; 46(3):612-7. http://dx.doi.org/10.1590/S0080-62342012000300012

8. Bardin L. Análise de conteúdo. São Paulo: Edições 70; 2011; p. 229

9. Bartolo M, De Luca D, Serrao M, Sinforiani E, Zucchella C, Sandrin G. Caregivers burden and needs in community neurorehabilitation. J Rehabil Med. 2010;42(9):818-22. http://dx.doi.org/10.2340/16501977-0612

10. Lopes RA, Mitre NCD, Coelho MAGM, Queiroz BZ. Perfil dos cuidadores das instituições de longa permanência para idosos de Itaúna - MG. ConScientiae Saúde 2012;11(2):338-44. http://dx.doi.org/10.5585/ConsSaude.v11n2.2985

11. Carli L, Kolankiewicz ACB, Loro MM, Rosanelli CLSP, Stumm EFM, Pettenon MK. Ambiente asilar: única opção de moradia. Revista Contexto e Saúde. 2011;11(20):647-52. http://dx.doi.org/10.21527/2176-7114.2011.20.647-652

12. Silva CA, Menezes MR, Santos ACPO, Carvalho LS, Barreiros EX Relacionamento de amizade na instituição asilar. Rev Gaúcha Enferm. 2006;27(2):274-83. 
13. Rissardo LK, Furlan MCR, Grandizolli G, Marcon SS, Carreira L. Sentimentos de residir em uma instituição de longa permanência: percepção de idosos asilados. Rev Enferm UERJ. 2012;20(3):380-5.

14. Mariano PP, Carreira L. Prazer e sofrimento no cuidado ao idoso em instituição de longa permanência: percepção dos trabalhadores de enfermagem. Esc Anna Nery. 2016; 20(4):e20160088. http://dx.doi.org/10.5935/1414-8145.20160088

15. Bessa MEP, Silva MJ, Borges CL, Moraes GLA, Freitas CASL. Idosas residentes em instituições de longa permanência: uso dos espaços na construção do cotidiano. Acta Paul Enferm. 2012:25(2):177-82.

http://dx.doi.org/10.1590/S0103-21002012000200004

16. Silva BT, Santos SSC, Silva MRS, Sousa LD. Percepção das pessoas idosas sobre a institucionalização: Reflexão acerca do cuidado de enfermagem. Rev Rene. 2009;10(4):118-25.

17. Castro VC, Derhun FM, Carreira L. Satisfação dos idosos e profissionais de enfermagem com o cuidado prestado em uma instituição asilar. Rev Pesqui Cuid Fundam. 2013;5(4):493-502.

18. Brasil. Agência Nacional de Vigilância Sanitária (Anvisa). Resolução RDC n. 283, de 26 de setembro de 2005. Aprova o Regulamento Técnico que define normas de funcionamento para as Instituições de Longa Permanência para Idosos. Diário Oficial União. Brasília: 2005.

19. Herédia VBM, Casara MB, Cortelletti IA. Impactos da longevidade na família multigeracional. Rev Bras Geriatr Gerontol. 2007;10(1):7-28.

20. Gvozd R, Dellaroza MSG. Velhice e a relação com idosos: o olhar de adolescentes do ensino fundamental. Rev Bras Geriatr Gerontol. 2012;15(2):295-304. http://dx.doi.org/10.1590/S1809-98232012000200012

21. Fonseca AC, Scoralick F, Piazzolla L, Palma D, Bertolino A, Silva $A$, et al. Perfil epidemiológico de idosos e fatores determinantes para a admissão em instituições de longa permanência no Distrito Federal. Brasília Med. 2011; 48(4):366-71.

22. Heredia VBM, Cortelletti IA, Cassara MB. Abandono na velhice. Textos Envelhec. 2005;8(3):307-19.

23. Leite SC, Marques IR. Sentimentos de idosos ao serem internados em instituição de longa permanência. Rev Enferm UNISA. 2011;12(1):31-7.

24. Karsch UM. Idosos dependentes: famílias e cuidadores. Cad Saúde Pública. 2003;19(3):861-6. http://dx.doi.org/10.1590/S0102-311X2003000300019
25. Creutzberg M, Gonçalves LHT, Sobottka EA. Instituição de Longa Permanência para idosos: a imagem que permanece. Texto Contexto Enferm. 2008;17(2): 273-9. http://dx.doi.org/10.1590/S0104-07072008000200008

26. Ocaña MRJ, Gomez AT, Ponce CR, Rodríguez JBR. Autonomía y estado de salud percibidos en ancianos institucionalizados. Gerokomos. 2006;17(1):6-23.

27. Cardona-Arango D, Estrada-Restrepo A, Chavarriaga-Maya LM, Segura-Cardona AM, Ordoñez-Molina J, Osorio-Gómez JJ. Apoyo social dignificante del adulto mayor institucionalizado. Rev Salud Pública. 2010;12(3):414-24.

28. Costa MCNS, Mercadante EF. O Idoso residente em ILPI (Instituição de Longa Permanência do Idoso) e o que isso representa para o sujeito idoso. Rev Kairós Gerontol. 2013;16(2):209-22.

29. Rocha FCV, Carvalho CMRG, Figueiredo MLF, Caldas CP. O cuidado do enfermeiro ao idoso na estratégia saúde da família. Rev Enferm UERJ. 2011;19(2):186-91.

30. Carneiro RS, Falcone E, Clark C, Prette ZD, Prette AD. Qualidade de vida, apoio social e depressão em idosos: relação com habilidades sociais. Psicol Reflexão Crit. 2007;20(2):229-37. http://dx.doi.org/10.1590/S0102-79722007000200008

31. Galindo D, Paiva AM. A construção de lugares no cotidiano de idosos em instituições totais: subsídios para a atuação em asilos. Interseção. 2008;1(2):21-30.

32. Sampaio AMO, Rodrigues FN, Pereira VG, Rodrigues SM, Dias CA. Cuidadores de idosos: percepção sobre o envelhecimento e sua influência sobre o ato de cuidar. Estud Pesqui Psicol. 2011:11(2):590-613.

33. Vagetti GC, Weinheimer MS, Oliveira V. Atendimento integral à saúde do idoso residente em instituição de longa permanência: uma experiência interdisciplinar. Estud Interdiscip Envelhec. 2007;11:53-66.

34. Santos SSC, Silva BT, Barlem ELD, Lopes RS. O papel do enfermeiro na instituição de longa permanência para idosos Rev Enferm UFPE. 2008; 2(3):291-9. http://dx.doi.org/10.5205/reuol.351-11415-1-LE.0203200812

35. Silva ILS, Machado FCA, Ferreira MAF, Rodrigues MP. Formação profissional de cuidadores de idosos atuantes em instituições de longa permanência. HOLOS. 2015;8: 342-56. http://dx.doi.org/10.15628/holos.2015.3215

36. Brasil. Ministério da Saúde. Estatuto do idoso. 2ed. rev. Brasília: Ministério da Saúde. 2009.

37. Fernandes $M$, Andrade A, Nóbrega M. Antecedents of frailty in the elderly: a systematic revision. Online Braz J Nurs. 2010;9(1). 